\title{
Amenazas Cibernéticas a la Seguridad y Defensa Nacional. Reflexiones y perspectivas en Colombia
}

\author{
M. E. Realpe y J. Cano ${ }^{2}$
}

\begin{abstract}
Resumen- Las amenazas cibernéticas impuestas por las tecnologías disruptivas en las Fuerzas de la Ley del Estado, sugieren una peligrosa tendencia hacia la Seguridad y Defensa Nacional. Para mitigar los riesgos en este campo, se requiere una estrategia integral para contrarrestar, si es necesario, la resistencia a los ataques disruptivos y destructivos. En efecto, este documento presenta un análisis general de la situación actual en el campo de la Ciberdefensa en Colombia, donde se identifican las ciberamenazas latentes y emergentes mediante el instrumento denominado Ventana AREM, enfrentando siete (7) tecnologías disruptivas analizadas por los investigadores del MIT a corto plazo, con el fin de proponer una estrategia militar de Ciberdefensa que permita responder a las ciberamenazas con una visión integral, sistémica y prospectiva.
\end{abstract}

Palabras clave - Ciberdefensa, Tecnologías disruptivas, Ciberamenazas, Estrategia.

\section{INTRODUCCIÓN}

$\mathrm{L}$ A CONVERGENCIA de tecnologías digitales, físicas y biológicas marcan el advenimiento y despliegue de la Cuarta Revolución Industrial o Revolución Digital, generando un cambio veloz y a gran escala en los sistemas informáticos que actualmente se conocen [23]. Este escenario genera un espacio propicio para la incubación de amenazas cibernéticas, que pueden poner en riesgo la prosperidad económica y social de un país, así como su Seguridad y Defensa Nacional.

Dichas amenazas cibernéticas son múltiples y muy variadas, mutan sus vectores de operación en cuestión de segundos, y se soportan en la masificación de las tecnologías disruptivas. Aunado a este comportamiento, los perpetradores de ataques (o adversarios) no descansan en su intento por buscar nuevas y mejores formas de explotación para lograr sus objetivos de manera más fácil y eficiente, creando un escenario complejo y dinámico que debe ser afrontado por las Fuerzas de Ley, de la forma más adecuada, tanto para anticipar como evitar que se produzca una afectación a la Seguridad Nacional. Se debe agregar que, como lo afirma el autor Schwab Klaus, la revolución digital en su esencia, "No cambia lo que hacemos, sino que cambia lo que somos" [22], lo que marca un inicio para repensar y evolucionar el concepto de la Defensa Nacional al nuevo entorno operacional llamado ciberespacio, con el fin de comprender la dinámica de las vulnerabilidades y los retos ante la nueva revolución digital. En este nuevo dominio, es posible que un ataque cibernético realizado a las

1. M. E. Realpe. Escuela Superior de Guerra, Colombia. Milena.realpe@esdegue.edu.co

2. J. Cano. Académico y profesor universitario, Colombia. jjcano@yahoo.com plataformas tecnológicas que soportan los servicios esenciales brindados a la población debilite o impida la gobernabilidad de un país, imposibilite la prestación de servicios esenciales ocasionando sufrimiento y, en otros casos, la muerte e incluso desequilibre la economía; hechos que en conjunto pueden desestabilizar la Seguridad y Defensa Nacional. En consecuencia, es necesario considerar el ciberespacio como un nuevo escenario de confrontación bélica, que combinado con los dominios de tierra, mar, aire y espacio constituirán una capacidad y superioridad relevante de cualquier Estado o Nación.

Con respecto a la evolución y el crecimiento tecnológico, es apropiado decir que éstos toman cada vez menos tiempo por lo que, para hablar del futuro del ciberespacio, este documento establece como referencia el año 2022, considerando base el estudio realizado por Instituto Tecnológico de Massachussets (MIT) [23] y las reflexiones de Klaus Schwab (2018), en su libro "La cuarta revolución industrial", donde se pronostica que "las innovaciones tecnológicas más importantes están a punto de generar un cambio trascendental en todo el mundo y esto es inevitable" [22]. En este sentido, este artículo plantea una estrategia de defensa cibernética a corto plazo para Colombia, de tal manera que, pueda ser adoptada para obtener victorias tempranas y contar con una ventana estratégica de acciones preventivas.

En consecuencia, la Estrategia Militar de Ciberdefensa para las Fuerzas Militares de Colombia que se plantea de cara a las amenazas cibernéticas latentes y emergentes que imponen las tecnologías disruptivas, parte de un análisis de capacidades existentes en las instituciones responsables de la Ciberdefensa Nacional de las Fuerzas Militares, a nivel de doctrina, organización, material, personal e infraestructura $u$ otras variables que permitan determinar la situación actual y así, proyectar un estado deseado. De igual forma, se realiza la identificación y estudio de las amenazas cibernéticas latentes y emergentes que imponen las tecnologías disruptivas a la Ciberdefensa Nacional en Colombia basado en el instrumento llamado la "Ventana de AREM" [13].

Finalmente, con los resultados obtenidos se presenta un esquema general de una propuesta militar para desarrollar capacidades cibernéticas en las Fuerzas Militares, fundamentada en el modelo de planeamiento por capacidades DOMPILEM (Doctrina, Organización, Material, Personal, Infraestructura, Liderazgo, Entrenamiento, Mantenimiento), que permitan configurar unas capacidades que permitan el despliegue operativo necesario para responder ante las nuevas amenazas cibernéticas. Por razones de confidencialidad, Seguridad y Defensa Nacional, este artículo solo presenta un 
esquema general de fortalecimiento cibernético y algunos contenidos necesarios para su comprensión.

\section{CONSIDERACIONES DE LA CIBERDEFENSA NACIONAL}

Hoy en día, la tecnología y el internet hacen parte integral en el desarrollo económico, político y social de Colombia y por ende de su Seguridad y Defensa Nacional. Es por esto, que la Ciberdefensa Nacional, debe ser asumida con un enfoque sistémico y multidimensional que requiere un trabajo conjunto y coordinado con los diferentes sectores e instituciones del país para dar cumplimiento a los objetivos e intereses del Estado.

En este sentido, sus Fuerzas Militares, requieren abordar el ciberespacio como un ámbito estratégico, operativo y táctico, para organizar, entrenar y equipar a sus hombres, con el fin de aplicar medidas de prevención, disuasión, contención, protección y reacción, que permitan fortalecer las capacidades de Ciberdefensa, para enfrentar las amenazas o ataques cibernéticos que puedan afectar la infraestructura crítica cibernética del país y poner en riesgo la seguridad nacional, la defensa de la soberanía y el orden constitucional del Estado, así como causar daños masivos, debilitar la economía, y/o dañar la moral pública y la confianza.

Para tal fin, el Ministro de Defensa Nacional de Colombia del año 2012, aprobó la creación y activación del Comando Conjunto Cibernético-CCOCI [21] con la función principal de ejercer la Ciberdefensa de la Nación y conducir operaciones militares cibernéticas a nivel estratégico, para ser garantes de la Seguridad y Defensa de la Nación en el ciberespacio. De igual forma, se ordenó la creación de estructuras organizacionales al interior de cada Fuerza denominadas Unidades Cibernéticas, así: una en el Ejército Nacional, otra en la Armada Nacional y una tercera en la Fuerza Aérea Colombiana, con las cuales el CCOCI ejecutará y coordinará actividades de Ciberseguridad y operaciones de Ciberdefensa del país.

En la actualidad, Colombia cuenta con una estructura organizacional en materia de Ciberdefensa en los niveles estratégico, operacional y táctico. De acuerdo con la primera edición del Manual de Ciberdefensa Conjunta del CCOCI [4], la estructura organizacional de Ciberdefensa se encuentra bajo la responsabilidad del Comando General de las Fuerzas Militares (COGFM) a través del Comando Conjunto Cibernético (CCOCI) que depende de la Subjefatura de Estado Mayor Conjunto Operacional (SEMCO) y de las Unidades Cibernéticas del Ejército Nacional, la Armada Nacional y la Fuerza Aérea Colombiana, de aquí en adelante las Fuerzas.

En efecto, el CCOCI fue activado en octubre de 2012 como ente rector para realizar el direccionamiento, planeación, coordinación, integración, ejecución y sincronización de Operaciones Cibernéticas. Dicho Comando, tiene la misión de ejercer la Ciberdefensa y conducir Operaciones Militares
Cibernéticas a nivel estratégico en el ciberespacio. Para tal fin, dicho Comando mantiene relaciones de coordinación con las Unidades Cibernéticas de las Fuerzas, con el propósito de consolidar esfuerzos e integrar capacidades para las Operaciones de Ciberseguridad y Ciberdefensa [18]. En el Ejército Nacional se creó el Grupo de Apoyo de Comunicaciones y Ciberdefensa, en la Armada Nacional, se creó la Dirección Cibernética Naval, en la Fuerza Aérea Colombiana se creó la Dirección Cibernética Aérea; así mismo de manera coordinada, conjunta y combinada realizan operaciones para la Defensa y Seguridad Nacional.

Adicionalmente, estas Unidades Militares lideran trabajos interinstitucionales en materia de Infraestructuras Críticas Cibernéticas, con el propósito de avanzar en el desarrollo de Políticas y Planes de Protección y Ciberseguridad para sus activos estratégicos nacionales. Como resultado, se han logrado establecer lazos de confianza y amistad entre los diferentes sectores del país que permitieron conformar redes de colaboración para compartir información de amenazas y alertas tempranas como medida preventiva para evitar la materialización de amenazas o ataques sobre las infraestructuras cibernéticas del país. Sin embargo, pese a los esfuerzos realizados, se evidencia la carencia de unas políticas y directrices integrales, positivas y comunes que describan y detallen las oportunidades y desafíos en el quinto dominio de la guerra, el ciberespacio, para empoderar a cada sector en materia cibernética, estableciendo objetivos estratégicos y líneas de acción claras que permitan configurar un control institucional en materia cibernética para conseguir los objetivos nacionales.

\section{AMENAZAS CIBERNÉTICAS DE CARA A LAS TECNOLOGÍAS DISRUPTIVAS}

La actualidad tecnológica exige un mundo de sistemas complejos e interconectados que traen como consecuencia el crecimiento de las amenazas cibernéticas a ritmos exponenciales y a velocidades desconcertantes. En este contexto, la Ciberdefensa toma un papel preponderante que involucra diversos actores, dándole el eje fundamental a las Fuerzas Militares, para proponer una visión que asegure la defensa cibernética nacional, así como la resiliencia y continuidad de las Infraestructuras Críticas Cibernéticas, en el quinto dominio de la guerra: el ciberespacio [5]. Por su parte, la convergencia tecnológica, la densidad digital, los productos y servicios digitalmente modificados, entre otros, han incrementado los niveles de riesgo cibernético nacional. En este contexto, se revela que estamos frente a una serie de retos que exigen altos niveles de dependencia tecnológica, que despliega un escenario de oportunidades, pero también complejo y desafiante de cara al crecimiento exponencial de las amenazas y vulnerabilidades cibernéticas a la Ciberdefensa Nacional [12].

En este sentido, Colombia está adoptando tecnologías de información y tecnologías de operación para optimizar el desarrollo de sus procesos productivos, económicos, 
sociales, políticos e incluso de Seguridad y Defensa Nacional. Dichos procesos se encuentran frente a un escenario de cambios disruptivos, en los cuales se hace necesario adaptarse y prepararse para anticipar y evitar (en la medida de lo posible) daños de alto impacto en las Infraestructuras Críticas Cibernéticas del país, que afecten directamente la prestación de los servicios básicos a la población y, por consiguiente, comprometan la prosperidad económica y social de la Nación. Es una realidad que un gran número de fuerzas de disrupción e innovación están marcando el futuro inmediato, pero también dan lugar al surgimiento de nuevas amenazas $y$ ataques en el ciberespacio que podrían llegar a afectar infraestructuras cibernéticas provocando incluso daños físicos. Por lo tanto, los perpetradores de ataques no descansan en su intento por buscar nuevas y mejores formas de explotación para lograr sus objetivos de manera más fácil, eficiente y anónima.

Para iniciar a hablar de tecnologías disruptivas es necesario empezar por definirlas: "Las tecnologías disruptivas son "tecnologías cuya aplicación rompe con los patrones que existía hasta el momento", apunta Carlos Martín, profesor del Master of Science in IT Strategic Management de la UPF Barcelona School of Management. Por su parte, Clayton M. Christensen, profesor de Harvard Business School, acuñó el término "tecnología disruptiva". En su libro de 1997, The Innovator's Dilemma, Christensen, separa la nueva tecnología en dos categorías: sostenida y disruptiva. La tecnología sostenida se basa en mejoras incrementales a una tecnología ya establecida. La tecnología disruptiva carece de refinamiento, a menudo tiene problemas de rendimiento porque es nueva, atrae a un público limitado y puede que aún no tenga una aplicación práctica probada (tal fue el caso de la "máquina de habla eléctrica" de Alexander Graham Bell, que ahora llamamos el teléfono).

Dicho de otra manera, el concepto de "Tecnologías Disruptivas", implica, entre otras cosas una revolución tecnológica, un cambio en la manera de hacer las cosas. Un estudio realizado por el Instituto tecnológico de Massachusetss (MIT), define siete tecnologías que cambiarán el mundo, las cuales serán objeto de estudio del presente documento: [23], así:

\section{Machine Learning (aprendizaje automático)}

De acuerdo con el estudio realizado por el MIT, existen 3 enfoques diferentes: a) Perspectiva de la computadora, programas que "aprenden", b) Una perspectiva relacional, abarca reconocimiento de patrones basado en computadora, modelado estadístico y análisis para la toma de decisiones, y c) Perspectiva holística, combina algoritmos informáticos, patrones estadísticos e inteligencia artificial. Adicionalmente, hay tres principios tecnológicos que, cuando se agrupan, desbloquean los aspectos clave de cada perspectiva: computación en la nube, grandes datos $\mathrm{y}$ analítica e inteligencia artificial. Cuando se analiza el
Machine Learning desde esta perspectiva se determina inofensiva y favorable, sin embargo, el futuro parece muy distinto cuando se analiza desde la Seguridad y Defensa Nacional. Es por esto, que se hace necesario cuestionar ¿Qué ocurriría si esta tecnología se despliega para llevar a cabo ataques cibernéticos contra las infraestructuras críticas nacionales?

\section{Nanotechnology (Nanotecnología):}

Es una ciencia de la ingeniería que está diseñando y fabricando circuitos y dispositivos extraordinariamente pequeños que se construyen a nivel molecular de materia, típicamente de 1 a 100 nanómetros. Las combinaciones de nanomateriales pueden marcar el comienzo de una nueva era que nos proporciona computadoras y otros dispositivos con un poder de procesamiento hasta ahora inalcanzable. Con la nanotecnología, todo tipo de medios y materiales que no han tenido capacidades de procesamiento $y$ entrega de información pueden convertirse en nuevos portales de comercio y comunicación. Los materiales de nanoingeniería adoptarán nuevas propiedades físicas que son muy diferentes y más útiles que las propiedades físicas de sus estados naturales. Esta tecnología, también se desarrolla en el límite de lo desconocido, y como tal se sujeta a la Seguridad y Defensa al umbral de la incertidumbre. Al manipular los materiales a esta escala, los riesgos pueden ser impredecibles e imperceptibles a los sentidos, incrementando los grados de complejidad en lo que refiere a afectaciones éticas, legales, ambientales y de salud entre otras para Colombia.

\section{Robótica}

La robótica no es una tecnología nueva en sí mismo, pero en la última década, la robótica ha sufrido una transformación radical impulsada por tres características: [23]

- Precisión: La capacidad de realizar tareas extremadamente exactas.

- Agilidad: La capacidad de realizar una variedad de tareas de forma rápida y fácil.

- Inteligencia: La capacidad de adquirir y aplicar nuevos conocimientos y habilidades.

Los robots son cada vez más utilizados en todos los sectores, en un sin número de tareas en educación, agricultura, salud, comercio, industria, turismo e incluso en la Seguridad y Defensa. Su rápida evolución pronto hará que la colaboración entre seres humanos y máquinas sea una realidad cotidiana que incremente la eficiencia, precisión y velocidad y reduzca los riesgos de tareas peligrosas minimizando los costos. Las personas que abogan por el desarrollo y despliegue de los sistemas de armamento autónomos normalmente resaltan varias ventajas militares. En primer lugar, los sistemas de armamento autónomos actúan como multiplicadores de Fuerza. Es decir, se necesita un menor número de soldados para cumplir una misión dada 
y se incrementa la eficacia de cada soldado. En segundo lugar, los defensores adscriben la expansión del campo de batalla a los sistemas de armamento autónomos, que permite que el combate alcance áreas que previamente eran inaccesibles. En tercer lugar, los sistemas de armamento autónomos pueden reducir el número de bajas al quitar a los combatientes humanos de las misiones peligrosas [18]. Cuando se analiza esta tecnología desde el polo opuesto a la beneficiosa es atinado pensar que las afectaciones para la Seguridad y Defensa de una Nación podrían ser demoledoras. ¿En quién podría recaer la responsabilidad cuando se despliegan los sistemas de armas que generan daños a la población, o cuando un sistema sea capaz de asumir su propio control?

\section{Impresión en tres dimensiones:}

Consiste en crear un objeto físico mediante la impresión capa por capa de un modelo o un dibujo en 3D. Con el tiempo las impresoras 3D superarán los obstáculos de velocidad, costo y tamaño y su uso será más generalizado. Esta tecnología ofrece ventajas y también varias desventajas, relacionadas a la creación propia de productos de forma automatizada mediante impresoras 3D. Entre las ventajas están la flexibilidad y prototipado rápido, reducción de costos, personalización, nueva industria, así como aplicaciones múltiples aún por descubrir. Para analizar las desventajas es preciso acoger una óptica desde la orilla de los malintencionados, donde existe la posibilidad de crear objetos tales como armas de fuego, drogas o elementos de alta peligrosidad que deja entrever el peligro de llegar a una proliferación de elementos y una falsificación sin límites de elementos que puedan ser usados en contra de las Fuerzas de Ley.

\section{Biotecnología}

Es el uso de sistemas vivos y organismos para desarrollar o fabricar productos. En la actualidad, los avances en tecnología digital, ingeniería genética, informática, tecnología celular y ciencias químicas están ampliando enormemente los límites de la biotecnología. La noción de la ingeniería de las células vivas y el surgimiento de la industria de las ciencias de la vida cambiará radicalmente los límites de la atención médica, la agricultura y los productos químicos. La biotecnología apunta a ser una de las áreas de mayor importancia en la sociedad, particularmente en el siglo XXI. Pese a las ventajas que aporta esta tecnología a la humanidad, también trae consigo una serie de riesgos, principalmente en el medio ambiente y en la salud como la modificación de la estructura del genoma humano, clonación y la manipulación del material genético de nuestra especie entre otros.

\section{Wireless mesh networks (Redes Inalámbricas)}

Las redes de malla inalámbricas (WMN) son circuitos ad hoc de conectividad inalámbrica en los que solo un dispositivo requiere una conexión a Internet. Estas son redes inteligentes de dispositivos inalámbricos que se pueden formar, dispersar y reformar según el comando del usuario. Las WMN se crean de abajo hacia arriba mediante conexiones entre dispositivos, sus capacidades de auto formación y auto curación aseguran una comunicación robusta y confiable en cualquier lugar a bajo costo y sin infraestructura fija. Las WMN amplían la informática generalizada integrada en el IoT (Internet of Things Internet de las Cosas) haciéndolo más dinámico. Esta tecnología ofrece múltiples beneficios, entre los más significativos están: la innovación y menor costo, al tiempo que los contras se consolidan en que no existe sistemas de regulación para controlar este tipo de tecnología y con ello se integran un sin número de delitos cibernéticos.

\section{Pervasive Computing (Computación Omnipresente}

También conocida como computación ubicua, la cual brinda información, medios, contexto y poder de procesamiento, sin importar la ubicación. Esta clase de tecnologías se caracteriza por amplias redes de microprocesadores conectados o incrustados en objetos cotidianos, los datos se integran y se intercambian en las redes públicas. La computación omnipresente es la tecnología que impulsa Internet de las cosas (IoT), pero es más preciso pensar en ella como el motor de todo el internet. Las capacidades de información, intercambio y colaboración de estas redes no se limitan a ningún dispositivo o ubicación fija; se distribuyen por todo el mundo. Además, el factor de forma de la informática dominante puede ser móvil, usable o implantable. El IoT como toda tecnología disruptiva, conlleva una serie de ventajas y de desventajas. Entre los riesgos más significativos que aporta esta tecnología a la Seguridad Nacional están: la falta de compatibilidad, la complejidad que representan y la falta de privacidad y seguridad, considerando que existe la posibilidad de que el software pueda ser vulnerado y los datos personales mal empleados.

En consecuencia, cada una de estas tecnologías marcarán un cambio profundo y sistémico en la sociedad, facilitarán los procesos y harán más eficiente la vida de los colombianos. Sin embargo, también traerán consigo novedosas amenazas y peligrosos ataques de tipo cibernético que pondrán en riesgo a la Nación.

\section{LA VENTANA DE AREM Y EL ANÁLISIS DE LAS AMENAZAS CIBERNÉTICAS}

En esta sección, se analizaron las amenazas cibernéticas haciendo uso del instrumento denominado "Ventana de AREM" [2], dado que, a diferencia de los marcos tradicionales de riesgos que se basan en riesgos y amenazas conocidas, ofrece una visión más amplia de éstos y las vulnerabilidades cibernéticas, con el fin de avanzar hacia una gestión y gobierno de los riesgos empresariales basado más en las posibilidades, que en las probabilidades. 
TABLA I

A pesar de que el autor plantea en su instrumento cuatro tipos de amenazas o riesgos así: Conocidos, focalizados, latentes y emergentes, para el caso que nos ocupa en el presente estudio se limitará al análisis de las amenazas y riegos latentes y emergentes que presentan las tecnologías disruptivas para Colombia a corto plazo, a fin de plantear una estrategia militar que permita dar respuesta y ser efectiva de cara a un análisis prospectivo a corto y mediano plazo.

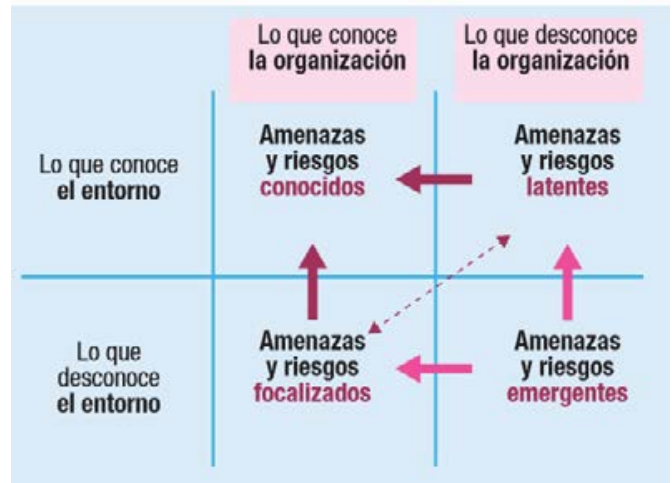

Figura 1. La Ventana de AREM [13].

En primer lugar, partiendo de las definiciones de amenaza latente (el analista se ha enterado que tal amenaza existe y no sabe si la organización tiene alguna estrategia de mitigación) y amenaza emergente (el analista nunca había escuchado de tal amenaza) [13], para aplicar este instrumento un analista de riesgos recolectó y analizó durante un tiempo (generalmente un año), material suficiente de tendencias visibles y emergentes sobre nuevas y posibles amenazas o vectores de ataque, las cuales se valoraron y priorizaron de acuerdo con su nivel de impacto en la organización, novedad del ataque y capacidad de respuesta actual de las Unidades de Ciberdefensa. Con base en este resultado, se estableció una lista de posibles amenazas y riesgos cibernéticos contrastadas con cada una de las siete tecnologías disruptivas mencionadas en la sección anterior, a fin de identificar las posibles amenazas que podrían afectar la Seguridad y Defensa Nacional en un futuro inmediato, las cuales fueron valoradas por los investigadores asistidos con la Ventana de AREM. Posteriormente se priorizaron basado en su impacto y compilaron los diferentes análisis, identificando las mayores coincidencias en las ubicaciones en los diferentes cuadrantes, para luego afinarse con el conocimiento del autor, en las realidades concretas del país y sus niveles de aseguramiento actuales.

El resultado, es una vista sistémica de los riesgos claves a tener en cuenta (dos cuadrantes), enriquecida con la vista de los investigadores y los analistas de riesgos y amenazas en el contexto de la Ciberdefensa Nacional, donde se obtuvo los resultados definidos en la tabla I:
AMENAZAS LATENTES Y EMERGENTES

\begin{tabular}{|c|c|}
\hline Amenaza Latente & Amenaza Emergente \\
\hline Ciberguerra & Guerra Autónoma \\
\hline LAWS Lethal Autonomous & Ciberarmas de destrucción \\
Weapons (Robots Militares, & masiva \\
embarcaciones de superficie & \\
y submarinas, drones & \\
autónomos, sistemas & \\
satelitales autónomos) & \\
\hline Sistemas Autónomos & \\
\hline
\end{tabular}

\section{Riesgos Latentes}

La Ciberguerra: es sin duda, el extremo más grave del espectro de los problemas de seguridad planteados en el ciberespacio donde los actores son los Estados. La ciberguerra puede permitir a los actores lograr sus objetivos políticos y estratégicos en menos tiempo, con mínimos riesgos y a bajo costo. La guerra cibernética o ciberconflicto se configura en la actualidad, como una posibilidad de intervención políticoestratégica más eficiente, en la que habrá más posibilidades de que los daños provocados sean menores en comparación con las armas convencionales. Además de eso, será también más difícil de identificar/controlar el origen de los ataques. Por tantas ventajas, las actividades que se suceden en el ciberespacio atraen el interés de los Estados, con el objetivo de utilizarlas como instrumentos de política exterior. El conflicto sigue siendo una extensión de la voluntad política y ahora tiene un nuevo dominio en el que sus formas pueden participar. Colombia, no se encuentra ajena a esta amenaza.

Las armas letales autónomas: (LAWS, por sus siglas en inglés, Lethal Autonomous Weapons). La ONU define como arma autónoma una herramienta capaz de "localizar, seleccionar y eliminar objetivos humanos sin intervención humana". Estas, son el resultado de la aplicar la inteligencia artificial a la búsqueda de soluciones en el ámbito del enfrentamiento militar; del combate. Las verdaderas armas autónomas letales o LAW, se enfrentarán al enemigo sin órdenes humanas y decidirán qué hacer para evitar los obstáculos que pretendan impedir la ejecución de su misión. Por ejemplo, un pequeño tanque podría patrullar las calles de una ciudad y actuar sin un piloto que decidiera por él. Estas opciones aun no existen, pero ya han desatado una gran polémica y un debate internacional. Hay LAW marinas o acuáticas (embarcaciones de superficie y submarinas), aéreas (drones autónomos que seleccionan por sí mismos los objetivos) y terrestres (algunos incluso con forma humana). El riesgo radica cuando sea la máquina mediante procedimientos autónomos la que elija los objetivos a abatir.

Sistemas Autónomos: Un reconocido científico y académico de la Universidad de California, llamado Stuart Russell, explica que los sistemas autónomos a su juicio es el problema actual de la inteligencia artificial. Ahora presente en un sinnúmero de dispositivos que podrían ser manipulados 
para infiltrar sistemas informáticos de otros Estados, empresas o industrias nacionales críticos para la sociedad sin intervención humana. La posible existencia de robots, capaces de disparar las 24 horas del día de manera indiscriminada: es uno de los escenarios que han dejado de pertenecer exclusivamente a las películas y series de ciencia ficción y se ha convertido en una preocupación recurrente de organismos internacionales como la ONU o la Convención de Ciertas Armas Convencionales. En los próximos años cada arma tendrá la capacidad de decidir autónomamente a quién matar: uno de los grandes dilemas éticos de la inteligencia artificial aplicada a la guerra. [9]

\section{Riesgos Emergentes:}

La Guerra Autónoma, es el uso de la inteligencia artificial en la guerra. Dicho de otra forma, una guerra autónoma acaba por transformarse en una guerra de recursos donde el humano no interfiere directamente. Simplemente es la interacción de Sistemas Autónomos entre sí, en este orden de ideas se hace necesario establecer pautas para la innovación, que impidan emprender un viaje tecnológico sin retorno.

Las Ciberarmas de Destrucción Masiva, si bien actualmente solo nueve Estados (supuestamente) poseen armas nucleares, las armas cibernéticas pueden ser obtenidas, desarrolladas o utilizadas por cualquier estado o actor no estatal; son relativamente baratos, seguros y fáciles de operar. Esto tiene dos consecuencias. Primero, las armas cibernéticas pueden convertirse en un nuevo tipo de Arma de Destrucción en Masa, o tal vez sería mejor llamarlas Armas de Destrucción Masiva. Es de esperar que dentro de unos años, gracias a la rápida $\mathrm{y}$ continua digitalización del mundo, los ciberataques puedan dañar a sociedades enteras. Es posible que las armas cibernéticas no puedan causar el mismo nivel de destrucción mortal que las armas nucleares, pero pueden ser muy eficaces. Piense en un sabotaje combinado y serio de la energía y los suministros de agua, así como en las comunicaciones [28].

Finalmente, y con base en los resultados obtenidos se propone contar con una Estrategia Militar basada en objetivos que consideran como fundamento el desarrollo de capacidades y la aplicación de medidas de contención y mitigación, los cuales sirvieron de base para la formulación de los objetivos estratégicos de la Estrategia propuesta.

\section{FORTALECIMIENTO DE LA CIBERDEFENSA NACIONAL}

La Estrategia Militar de Ciberdefensa asegura un enfoque conjunto, integral y sistémico que permita integrar los diferentes frentes y aristas relacionadas con la Ciberseguridad y Ciberdefensa Nacional para mejorar la eficacia operacional en el ciberespacio, proporcionando principios fundamentales que guíen el empleo de las Unidades de las Fuerzas Militares de Colombia (FF.MM.) hacia un objetivo común. Es necesario aclarar que las Fuerzas Militares de Colombia es el término usado para denotar colectivamente todos los componentes del Ejército, Armada y Fuerza Aérea [4].

Se resalta entonces, que a pesar de que la moderna tecnología ha revolucionado la mayor parte de las dimensiones materiales de la guerra desde el siglo XIX, la lógica de los conflictos permanece básicamente inalterable. Esto explica porque obras como "De la Guerra" de Carl Von Clausewitz [16] y "El Arte de la Guerra" de Sun Tzu [25] permanecen como relevantes marcos conceptuales para el estudio de la política y la estrategia incluso en nuestros días. Por lo tanto, a pesar de que la Estrategia Militar es una disciplina en constante evolución, la estrategia propuesta no puede ni debe ignorar las enseñanzas de los clásicos y debe contemplar los principios de la guerra a los que se acogen las Fuerzas Militares de Colombia, los cuales se encuentran enunciados en el Manual Fundamental de Referencia del Ejército MFRE 3-0 enfocadas para su empleo en el ámbito conjunto. La aplicación de estos principios clásicos en la conducción de Operaciones Conjuntas se define y amplía en el Manual de Operaciones Conjuntas. Adicionalmente se considera el modelo de guerra tradicional, donde los Estados-Nación luchan entre sí por razones tan variadas como la gama completa de sus intereses nacionales.

Las Operaciones Militares en la guerra tradicional normalmente se centran en las Fuerzas Militares de un adversario para influir finalmente en el Gobierno de éste. Con el caso cada vez más raro de guerra formalmente declarada, la guerra tradicional típicamente involucra operaciones militares Fuerza-a-Fuerza, en las cuales los adversarios emplean una variedad de fuerzas convencionales y fuerzas de operaciones especiales entre sí en todos los dominios físicos (incluyendo el ciberespacio). Un dominio es el territorio sobre el cual se impone o se ejerce el control (terrestre, marítimo, aéreo, espacial y el ciberespacio) [7]. Bajo este enfoque, y con base en los niveles de la guerra ${ }^{2}$, se definió y clarificó la relación entre los objetivos nacionales, el enfoque operacional y las tareas tácticas, considerando que no hay demarcaciones o límites definidos entre estos niveles, pero que ayudan a los comandantes a diseñar y sincronizar operaciones, asignar recursos y tareas al comando apropiado. En otras palabras, el propósito estratégico, operacional o táctico del empleo depende de la naturaleza del objetivo, la misión o la tarea [17].

Es por esto, que la Estrategia de Ciberdefensa propuesta se centra en la construcción y fortalecimiento de capacidades para el desarrollo de Operaciones Cibernéticas para operar en un dominio donde se disputa el acceso al ciberespacio o donde podría ser imperativo afectar el acceso a las comunicaciones contra cualquier adversario que intenta hacer daño a los intereses nacionales en tiempos de paz, crisis o conflicto para impedir los ataques y hacer las defensa del país, dando cumplimiento a las funciones asignadas al CCOCI

\footnotetext{
${ }^{2}$ Niveles de la Guerra: a) Estratégico nacional, b) Teatro estratégico, c) Operacional y d) Táctico
} 
mediante el CONPES 3701 "Lineamientos de Política para Ciberseguridad y Ciberdefensa del año 2011[10].

Mediante esta estrategia se propone planificar, desarrollar y utilizar las capacidades cibernéticas de las Fuerzas Militares de manera efectiva, y asegurar que las Operaciones en el ciberespacio se produzcan de manera consistente con los valores que promueve Colombia dentro de los límites que establece a la Fuerzas Militares, la legalidad y legitimidad a nivel nacional como internacional. En pocas palabras, se proporcionará dirección, coherencia y lineamientos con un enfoque integral para el desarrollo de capacidades militares en el ciberespacio en los próximos años. Para este fin se plantearon cinco (5) objetivos estratégicos, así:

Objetivo Estratégico 1 (OE1): Fortalecer el pie de Fuerza requerido para dar respuesta a las amenazas cibernéticas.

Objetivo Estratégico 2 (OE2): Defender las Infraestructuras Críticas Cibernéticas Nacionales de la actividad cibernética maliciosa.

Objetivo Estratégico 3 (OE3): Desarrollar capacidades cibernéticas para mejorar las ventajas militares en Colombia.

Objetivo Estratégico 4 (OE4): Conformar Ligas de Ciberdefensa con los sectores público y privado.

Objetivo Estratégico 5 (OE5): generar Alianzas Estratégicas a nivel nacional e internacional.

Estos objetivos estratégicos se analizaron contrastados con los componentes de capacidad abreviados en la sigla DOMPILEM (que será detallada más adelante). Estos componentes son aquellos aspectos que deben observarse en el planeamiento por capacidades, tanto en las soluciones materiales como en las no materiales, ante los requerimientos de la Fuerza para afrontar los retos presentes y futuros. Esta sigla también es empleada en la OTAN con una "I" adicional al final para indicar interoperabilidad, o con una "P" para incluir aquellas políticas gubernamentales o institucionales que afectan la capacidad [7].

La doctrina es generalmente el primer aspecto, ya que a menudo es el más fácil y rápido de actualizar y puede afectar drásticamente el desarrollo de las operaciones. En algunos casos, el impacto de los cambios en los otros componentes no puede realizarse plenamente sin alterar de manera significativa la doctrina. Además, la doctrina también puede servir como base para la evolución en las otras categorías DOMPILEM, con el fin de establecer los planes y tareas necesarias para alcanzar las metas: [7]

Doctrina: cómo se combate.

Organización: cómo es el diseño de la fuerza.

Material: elementos necesarios para equipar las fuerzas con el fin de que puedan operar de manera efectiva.

Personal: recurso humano necesario para combatir en la guerra, enfrentar contingencias o participar en operaciones de paz.

Instalaciones: bienes inmuebles.
Liderazgo y educación: cómo preparar a los comandantes en cada uno de los escalones para conducir el combate a través del desarrollo profesional.

Entrenamiento: cómo debe ser la preparación para el combate desde la formación básica hasta la formación individual de especialistas y el entrenamiento en los diferentes escalones. Mantenimiento: actividades que se requieren para el sostenimiento de la capacidad en el tiempo.

Con el propósito de identificar con claridad los componentes necesarios para alcanzar los objetivos estratégicos propuestos y con esto garantizar el cumplimiento de la Estrategia Militar de Ciberdefensa, se hizo necesario realizar un análisis DOFA (Debilidades, Oportunidades, Fortalezas, amenazas) que permitiera tener una visión global de la situación real de las Unidades de Ciberdefensa de las Fuerzas Militares y consecuentemente con los resultados obtenidos definir las necesidades para cada uno de los componentes. Posteriormente, y con base en los requerimientos más significativos analizados en cada matriz, se definió la matriz principal que refleja los componentes más significativos en cada uno de los objetivos estratégicos, así:

TABLA II RESUMEN ANALISIS OBJETIVOS VS DOMPILEM

\begin{tabular}{|c|c|c|c|c|c|c|c|c|}
\hline ORJ. & $\underset{\text { Dactrina }}{D}$ & $\begin{array}{c}o \\
\text { Organitación }\end{array}$ & $\begin{array}{c}M \\
\text { Material }\end{array}$ & $\begin{array}{c}P \\
\text { Persanal }\end{array}$ & $\begin{array}{c}I \\
\text { Instalaciones }\end{array}$ & $\begin{array}{c}L \\
\text { Lideraugo }\end{array}$ & $\begin{array}{c}E \\
\text { Entrenamiento }\end{array}$ & $\begin{array}{c}M \\
\text { Mantenimiento }\end{array}$ \\
\hline$O E t$ & $\begin{array}{c}\text { Manuales } \\
\text { conjuatosy } \\
\text { por Fuerza }\end{array}$ & $\begin{array}{c}\text { Un nuevio } \\
\text { componetele } \\
\text { minitur }\end{array}$ & $\begin{array}{l}\text { Haudraze } \\
\text { Solvare }\end{array}$ & $\begin{array}{l}\text { Masitury } \\
\text { Civil }\end{array}$ & $\begin{array}{c}\text { Oficmas } \\
\text { Luboratroios } \\
\text { Stens }\end{array}$ & 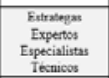 & Pesustente & $\begin{array}{l}\text { Plathformas } \\
\text { Ciberneticas }\end{array}$ \\
\hline $\mathrm{OE}_{2}$ & $\begin{array}{l}\text { Planes de } \\
\text { Protescion }\end{array}$ & $\begin{array}{c}\text { Equipsos } \\
\text { miterductuplanatios }\end{array}$ & $\begin{array}{l}\text { Platakinmas } \\
\text { compurtides }\end{array}$ & $\begin{array}{c}\text { Maitar } y \\
\text { Crual }\end{array}$ & $\begin{array}{l}\text { Propias y de } \\
\text { terceos }\end{array}$ & 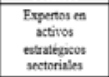 & \begin{tabular}{|c|} 
Sistemas Control \\
Industrial \\
II \\
To
\end{tabular} & $\begin{array}{c}\text { Sitrems de } \\
\text { Definas } \\
\text { Cibenética }\end{array}$ \\
\hline$O E_{3}$ & 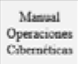 & $\begin{array}{l}\text { Equipos de } \\
\text { Bratlata }\end{array}$ & $\begin{array}{l}\text { Equipo de } \\
\text { combate } \\
\text { cibernetico }\end{array}$ & $\begin{array}{l}\text { Comandos } \\
\text { Cibemetios }\end{array}$ & \begin{tabular}{|c|} 
Sala Mando $y$ \\
Control \\
eberniticos
\end{tabular} & $\begin{array}{l}\text { Plan de Carren } \\
\text { Cibernitica }\end{array}$ & $\begin{array}{l}\text { Simulacicas } \\
\text { Olimpindas } \\
\text { Ciberoticas }\end{array}$ & 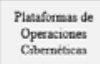 \\
\hline$O E_{4}$ & $\begin{array}{l}\text { Mammal de } \\
\text { Appoyo } \\
\text { Cibtenticos }\end{array}$ & $\begin{array}{l}\text { Confoumacióta } \\
\text { Ligas }\end{array}$ & $\begin{array}{l}\text { Eupesializado } \\
\text { Alto Nriel }\end{array}$ & $\begin{array}{l}\text { Sector } \\
\text { Pisticony } \\
\text { Priveds. }\end{array}$ & $\begin{array}{c}\text { Salus de } \\
\text { Intererembio } \\
\text { Cibetenticos }\end{array}$ & $\begin{array}{l}\text { Expetos } \\
\text { Ciberneticoss }\end{array}$ & \begin{tabular}{c|} 
Entresmiento \\
Interinstitucional
\end{tabular} & $\begin{array}{l}\text { Sustemas de } \\
\text { Apoyo }\end{array}$ \\
\hline$O E_{5}$ & $\begin{array}{l}\text { Acuedostor, } \\
\text { cosenenios }\end{array}$ & $\begin{array}{c}\text { Nacionales } \\
\text { Intermacionsles }\end{array}$ & $\begin{array}{l}\text { Especializado } \\
\text { Alto Nrel }\end{array}$ & \begin{tabular}{|c} 
Necionale \\
Intemacican!
\end{tabular} & $\begin{array}{l}\text { Propia y de } \\
\text { Terceros }\end{array}$ & Internacioanlistas & $\begin{array}{c}\text { Cuross } \\
\text { Intemaciventes }\end{array}$ & $\begin{array}{l}\text { Sustemas de } \\
\text { lesercambio }\end{array}$ \\
\hline
\end{tabular}

Esta tabla, refleja los elementos clave que permitirán que las Unidades de Ciberdefensa de las Fuerzas Militares, fortalezcan el desarrollo de capacidades para desarrollar operaciones militares cibernéticas efectivas para fortalecer la seguridad y defensa del país en el ciberespacio.

\section{CONCLUSIONES}

El ciberespacio es, por naturaleza, no es un espacio seguro y protegido, y, por tanto, es vulnerable a las amenazas o ataques cibernéticos latentes o emergentes, lo que puede resultar en pérdidas significativas para los sectores económico, político, social y/o constituir una seria amenaza para la defensa nacional o los intereses nacionales, por lo tanto el desarrollo de capacidades en el ciberespacio se constituye en una prioridad para la Seguridad y Defensa de Colombia, que cada vez más, es dependiente de la tecnología, al tiempo que el despliegue de operaciones militares en el ciberespacio es una necesidad para el avance de los modelos de defensa actuales. 
Para tener éxito y resultados verificables en las misiones cibernéticas que aseguren la Seguridad y Defensa Nacional, se hace necesario que las Fuerzas Militares realicen operaciones conjuntas entre las diferentes Fuerzas, coordinadas con la Policía Nacional, interinstitucionales con organizaciones tanto del sector público como privado y combinado con las naciones amigas, trabajando de la mano con los organismos binacionales y multilaterales, apoyados siempre que se requiera por las Ligas de Ciberdefensa.

La propuesta de la Estrategia Militar de Ciberdefensa es una respuesta efectiva a los riesgos y amenazas a los que se ve enfrentada la Seguridad y Defensa del país de cara a las tecnologías disruptivas, con ello, se planteó un modelo sistémico basado en objetivos estratégicos analizados en cada uno de los componentes del Modelo DOMPILEN. Con esto fue preciso delimitar y definir prospectivamente hacia donde deben ir las Fuerzas Militares a fin de desarrollar capacidades militares para el desarrollo de Operaciones Cibernéticas, soportadas en un marco legal y constitucional.

\section{REFERENCIAS}

[1] Accenture. (2015). Guiding digital transformation, 16.

[2] William C. (s.f.). Impact of Alleged Russian Cyber Attacks. Tomado https://nsarchive2.gwu.edu/NSAEBB/NSAEBB424/docs/ Cyber-027.pdf

[3] Clarke, J. and K. R. (2011). Guerra en la Red (1 edición).

[4] COGFM. (2018). Manual Fundamental Conjunto MFC 1.0 .

[5] DoD. (2018). National Cyber USA, (September).

[6] DoD Bulgaria. (2010). White paper on Defence and the armed forces of the Republic of Bulgaria, 71.

[7] EJC. (2017). Manual Fundamental Doctrina. (Centro de Doctrina del Ejército - CEDOE, Ed.) (Vol. 1). Bogotá: Imprenta Militar del Ejército Restricciones. Tomado de www.cedoe.mil.co

[8] Vergara GD, E. (2017). Operaciones militares cibernéticas. Buenos Aires.

[9] Guerrero, J. (n.d.). Armas autónomas, la amenaza

fantasma. Recuperado June 17, 2019, de

https://www.muyinteresante.es/revista-muy/noticias-

muy/articulo/armas-autonomas-la-amenaza-fantasma461487840117

[10] Gobierno Colombia (2017). CONPES 3701, 91. Recuperado https://colaboracion.dnp.gov.co/CDT/Conpes/Económicos/ 3701.pdf. Gobierno de Colombia.

[11] J, R. (2013). Political Guidance for Cyber Defence Portugal, 31976-31979.

[12] Cano, J. (2018). IT-Insecurity: Pronósticos de seguridad de la información 2018. Recuperado Feb 1, 2018, de http://insecurityit.blogspot.com.co/2017/10/pronosticosde-seguridad-de-la.html

[13] Cano, J. (2017) La ventana de AREM. Una estrategia para anticipar los riesgos y amenazas en ciberseguridad empresarial. ISACA Journal. 5. Recuperado de:
https://www.isaca.org/Journal/archives/2017/Volume-

5/Pages/the-arem-window-spanish.aspx

[14] Cano, J. (2018) Ciberdefensa Empresarial: Un marco conceptual y práctico en un entorno digitalmente inestable. En García, P., Barragán, R. \& Fuentes, N. M. (2018) Actas XV Reunión Española de Criptología y Seguridad de la Información. 96-101. ISBN: 978-84-09-02463-6. Recuperado de: https://www.researchgate.net/publication/328191823_Cibe rdefensa_empresarial_Un_marco_conceptual_y_practico_ en_un_entorno_digitalmente_inestable From: https://www.researchgate.net/publication/328191823.

[15] Aguilar, J. (2017). Industria 4.0: la cuarta revolución industrial. (S.A. MARCOMBO, Ed.). Marcombo. Retrieved from https://www.casadellibro.com/libroindustria-40-la-cuarta-revolucionindustrial/9788426725684/5991036

[16] Clausewitz, K. (2010). De la Guerra, 239. Recuperado de http://www.biblioteca.org.ar/libros/153741.pdf

[17] MFC1.0 (2018). Manual Fundamental Conjunto 1.0 Manual Reservado Comando General Fuerzas Militares.

[18] MCC (2016). Manual Ciberdefensa Conjunto Fuerzas Militares. Manual Reservado.

[19] Marchant, Allenby, Arkin, Barrett, Borenstein, Gaudet, , ... Meara, (2011). Science and Technology Law.

[20] Netherlands Ministry of Defence. (2012). The Netherlands - The defence cyber strategy, 20. Recuperado de http://www.defensie.nl

[21] Resolución Ministerial No. 7436 de 2012. Ministerio de Defensa de Colombia Documento Reservado.

[22] Klaus S. (2018). The Fourth Industrial Revolution, by Klaus Schwab | World Economic Forum. Recuperado marzo 11, 2018, de https://www.weforum.org/about/thefourth-industrial-revolution-by-klaus-schwab

[23] Segars, A. H. (2018). Seven Technologies Remaking the World. MIT Sloan Management Review, (March).

[24] Strategy Department Defence. (2014). Cyber Security Strategy for Defence, 1-18. Recuperado de https://ccdcoe.org/sites/default/files/strategy/Belgian Defence Cyber Security Strategy.pdf

[25] Sun Tzu. (2003). El Arte de la Guerra. Retrieved from http://www.biblioteca.org.ar/libros/656228.pdf

[26] The Department of Defense DoD of the United States of America. (2015). Strategic Cyber Defense.

[27] The Department of Defense DoD of the United States of America (2018). Strategic Cyber Defense. [2] Achtert, E. et al. Global Correlation Clustering Based on the Hough Transform. Statistical Analysis and Data Mining. vol 1(3), pp. 111-127. 2008

[28] Van Der Meer, S. (2016). Cyber Warfare and Nuclear Weapons: Game-changing Consequences?, (Dic), 36-38. Recuperado de http://www.techworld.com/news/security/cambridgeresearchers. 
Milena Elizabeth Realpe Diaz Oficial del Ejército Nacional, de profesión Ingeniera de Sistemas con énfasis en Telecomunicaciones, especialista en Seguridad de Redes de Computadores, en Seguridad Física y de la Informática, así como en Seguridad de la Información. Es Magister en Seguridad de la Información por la Universidad de los Andes y actualmente estudiante de la Maestría en Ciberseguridad y Ciberdefensa de la Escuela Superior de Guerra en Colombia.

Jeimy José Cano Martinez Ph.D, CFE. Profesor universitario a nivel pregrado, especialización, maestría y doctorado en Colombia y varios países de Latinoamérica en temas como computación forense, evidencia digital, gobierno y gestión de la seguridad de la información, gerencia de sistemas de información, criminalidad informática, auditoría de TI, ciberseguridad y protección de datos personales desde 1996 a la fecha. 